\title{
Measurement of Grain Size Distributions
}

\author{
George Vander Voort ${ }^{1}$, Olga Kurochkina ${ }^{2}$ and Alexander Kazakov²
}

${ }^{1}$ Consultant - Struers Inc., Wadsworth, Illinois

${ }^{2}$ St. Petersburg State Polytechnical University, St. Petersburg, Russia

ASTM standard E1181 discusses the characterization of duplex grain size microstructures and establishes a number of category types. There are various ways to characterize grain structures, including both manual and automatic image analysis approaches based upon either lineal or areal analysis of the grains. The word "duplex" implies that the structure is divided into two parts and may not really be the best term in dealing with grain size distributions. E1181 does not define what a "normal" grain size distribution is, nor does any other ASTM grain size standard. Perhaps this should be the starting point before dealing with deviations from a normal or Gaussian distribution. The ASTM grain size number scale is not linear, but is based on a power series. So, for a specimen with a uniform distribution of grain areas, a plot of grain area versus the area \% per class has a log normal distribution while a plot of the equivalent ASTM grain size class versus the area \% of grains per class will exhibit a normal distribution.

E1181 defines two basic types of duplex grain size distribution: random and topological in sections 8.3.1 and 8.3.2, respectively. There are three types of random duplex grain size distributions: the ALA (as large as), the wide range and the bimodal. An ALA type has a random distribution of individual coarse grains, differing in size by 3 or more ASTM grain size numbers from the average size and these individual coarse grains should comprise $5 \%$ or less of the area of the specimen. But, the "area of the specimen" is not defined. Does it mean the area of a 100X micrograph or the entire area of the polished surface? The wide-range duplex type is defined as an unusually wide range of grain sizes randomly distributed where the largest size differs from the smallest size grain by 5 or more ASTM grain size numbers. The bimodal type is defined as two distinct grain size populations, randomly distributed, such that the sizes differ by more than 4 grain size numbers, and that the two size populations together comprise $75 \%$ or more of the total surface area. What is the other $25 \%$ or less? The topological duplex conditions relate to a systematic variation in grain size across the section product form where the average grain sizes differ by 3 or more grain size numbers - called a "cross-section condition." A variation is the "necklace type" where individual coarse grains are surrounded by finer grains differing in size by 3 or more grain size numbers. The final type of topological duplex condition is called a "banded condition" where there are distinct bands with mean grain sizes differing by 3 or more grain size numbers.

An analysis of the nature of the grain size distribution is easiest determined and evaluated by measuring the area of each grain in the field of view. Next, the individual grain areas are aligned in decreasing size and the sum of the grain areas per ASTM G number is determined. For example, the areas of all grains 
with an area between that for a G of $3.5\left(11,405 \mu \mathrm{m}^{2}\right.$ ) and a G of 4.5 (area of $5703 \mu \mathrm{m}^{2}$ ) would be added together and classed as grain size 4 . This is repeated for all of the $G$ classes present in the specimen. Next, the area \% for each G class is calculated. A plot of $G$ versus the area \% per G class is constructed. The average grain size can be calculated from all of the measurements, particularly if the distribution is uni-modal. This work must start with evaluation of uni-modal grain size distribution specimens first, as shown in Fig. 1. Note that it covers 8 grain size classes and is, according to E1181, a duplex wide range condition. Figure 2 shows the microstructure of 330 austenitic stainless steel covering $9 \mathrm{G}$ classes. It does exhibit a wide-range "duplex" condition. But, is this a "duplex" condition? The E1181 classifications of non-normal grain size distributions must be re-evaluated using this approach.

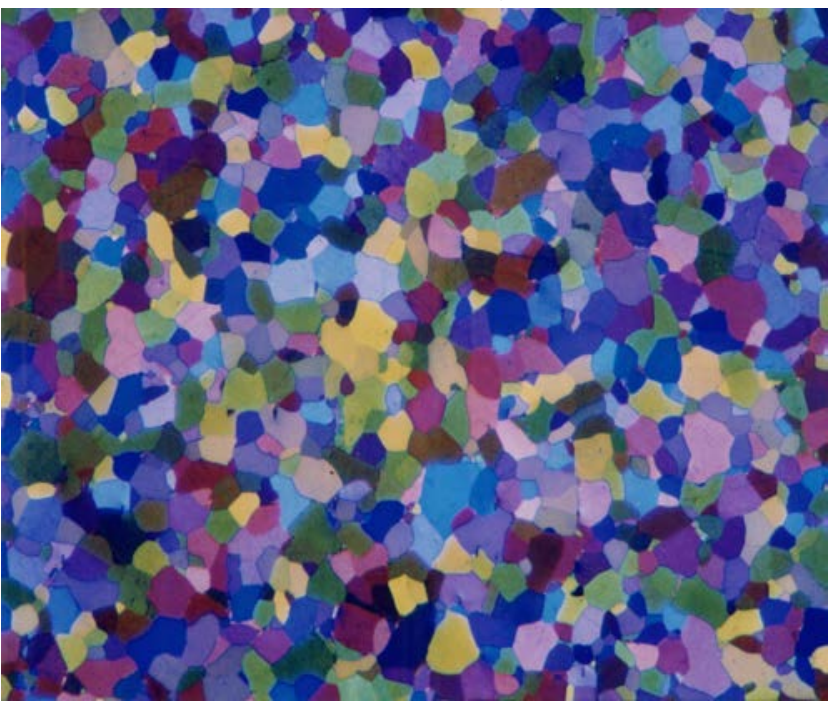

a) Motor Lamination Steel (original 100X)

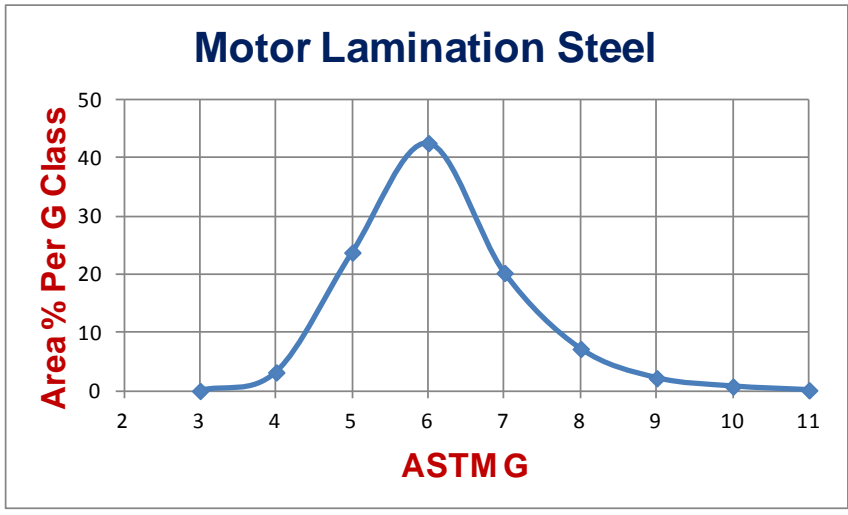

b) Normal grain size distribution

Figure 1. Grain size analysis of a steel with a uniform grain size distribution covering 8 grain size classes (no grains at $\mathrm{G}=3$ ) with a skew of 1.43 and a kurtosis of 2.55 (891 grains measured).

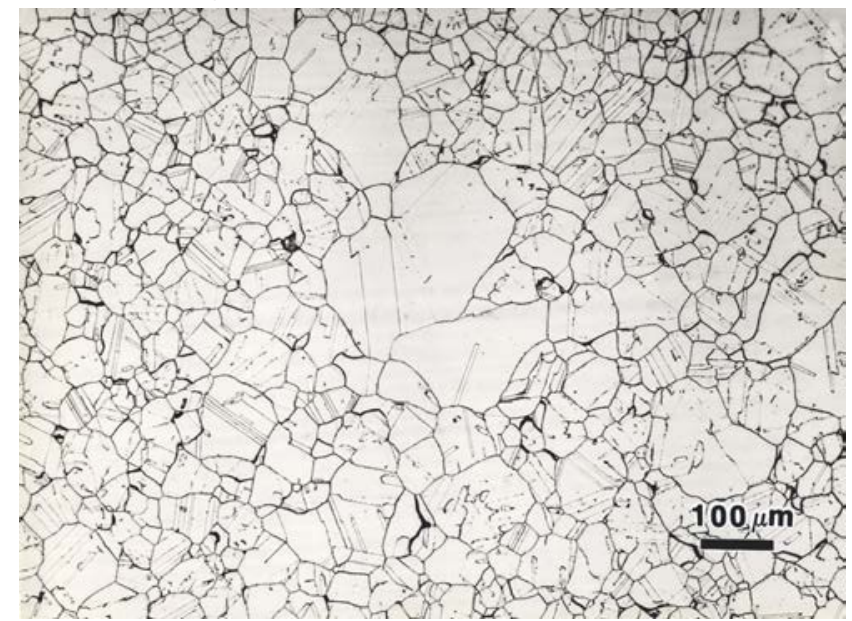

a) RA 330 Austenitic Stainless Steel

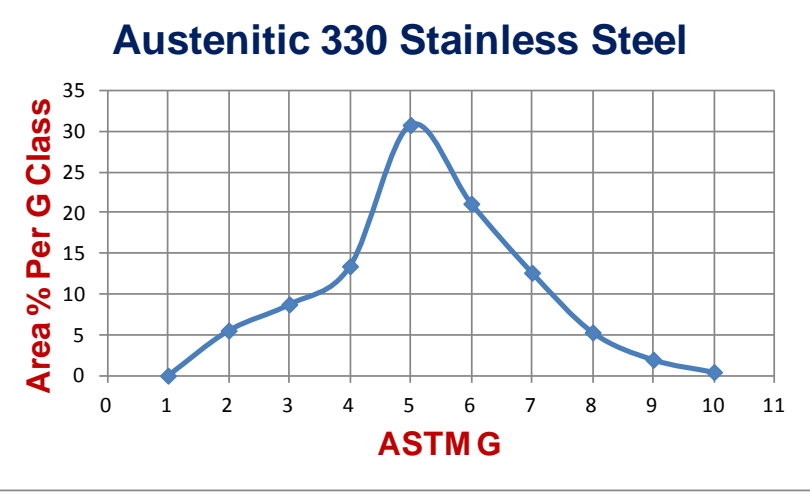

b) Wide range grain size distribution

Figure 2. Grain size analysis of a steel with a wide-range grain size distribution covering 9 grain size classes (no grains at G-1), a skew of 7.58 and a kurtosis of 86.88 (416 grains measured). 